\title{
THE SCHUR INDEX AND ROOTS OF UNITY
}

\author{
G. J. JANUSZ
}

\begin{abstract}
A short proof is given for the main step in the proof of the theorem of Benard and Schacher which asserts that if the Schur index of a character $\chi$ of a finite group is $m$ then the $m$ th roots of unity lie in the field of values $Q(\chi)$.
\end{abstract}

One of the most elegant results about the Schur index of a character of a finite group is the recent one by M. Benard and M. Schacher. They prove the Schur index over the rationals of the character $\chi$ can be $m$ only when the field of values of $\chi$ contains the $m$ th roots of unity. Their proof uses the Witt-Brauer reduction to the case of a "special" character, the theory of Hasse invariants and a rather long computational theorem of C. Ford [1]. We give here a proof of Ford's result (including a case not covered by him) in a version which covers the bulk of the BenardSchacher result. The proof uses only the most elementary facts about crossed products and the Brauer group. In order to make the proof even shorter, we shall state it as a result about algebras. For the passage from characters to special characters and then to algebras, the reader may consult any of the references listed.

Let $K$ be a field. A cyclotomic algebra over $K$ is a crossed product $\Delta=(K(\varepsilon), G, \beta)=\sum_{\sigma \in G} K(\varepsilon) u_{\sigma}$ in which $\varepsilon$ is a root of unity, $G$ is the Galois group of $K(\varepsilon)$ over $K$, and $\beta$ is a factor set whose values are roots of unity in $K(\varepsilon)$. The multiplication in $\Delta$ is given by the rules

$$
u_{\sigma} x=\sigma(x) u_{\sigma}, \quad u_{\sigma} u_{\tau}=\beta(\sigma, \tau) u_{\sigma \tau}
$$

for all $x$ in $K(\varepsilon)$ and $\sigma, \tau$ in $G$.

THEOREM. If the cyclotomic algebra $\Delta$ has exponent $m$ in the Brauer group of $K$, then the mith roots of unity lie in the center $K$.

Proof. We shall change factor sets in the proof so we write $\Delta(\beta)$ for the crossed product made with $K(\varepsilon), G$ and $\beta$. Let the values of $\beta$ generate a group $\left\langle\varepsilon_{n}\right\rangle$ of $n$th roots of unity. In the Brauer group of $K$, we have

$$
[\Delta(\beta)]^{n}=\left[\Delta\left(\beta^{n}\right)\right]=[\Delta(1)]=1 .
$$

Received by the editors February 7, 1972.

AMS 1970 subject classifications. Primary 13A20, 20C15.

Key words and phrases. Crossed product, Schur index, Brauer group.

(c) American Mathematical Society 1972 
By assumption the order of $[\Delta(\beta)]$ is $m$ so $m$ divides $n$. Thus a primitive $m$ th root $\varepsilon_{m}$ lies in $K(\varepsilon)$ and it is necessary to show it lies in $K$. We show this by proving $\varepsilon_{m}$ is fixed by all elements in $G$. Let $\tau \in G$ and let $\Delta\left(\beta^{r}\right)$ denote the crossed product $\left(K(\varepsilon), G, \beta^{r}\right)=\sum_{\sigma \in G} K(\varepsilon) v_{\sigma}$, where the new factor set $\beta^{\tau}$ is obtained by applying $\tau$ to the factor set $\beta$. It is necessary to check that $\beta^{r}$ is indeed a factor set. This is not in general true but works here because $G$ is abelian. The verification is left to the reader. Now map $\Delta(\beta)$ to $\Delta\left(\beta^{r}\right)$ by $\sum x_{\sigma} u_{\sigma} \rightarrow \sum \tau\left(x_{\sigma}\right) v_{\sigma}$. One may now check this is a $K$ algebra isomorphism and so $[\Delta(\beta)]=\left[\Delta\left(\beta^{r}\right)\right]$ in the Brauer group of $K$. Now there is a positive integer $r$ such that $\tau\left(\varepsilon_{n}\right)=\left(\varepsilon_{n}\right)^{r}$. It follows that $\beta^{r}=\beta^{r}$. Thus

$$
[\Delta(\beta)]=\left[\Delta\left(\beta^{r}\right)\right]=[\Delta(\beta)]^{r} .
$$

Since $[\Delta(\beta)]$ has order $m$ we find $m$ divides $r-1$; or $r=1+m k$. Now $\varepsilon_{m}$ lies in $\left\langle\varepsilon_{n}\right\rangle$ so $\tau\left(\varepsilon_{m}\right)=\left(\varepsilon_{m}\right)^{r}=\left(\varepsilon_{m}\right)^{1+m k}=\varepsilon_{m}$ which proves $\varepsilon_{m}$ is fixed by all elements of $G$ as required.

\section{REFERENCES}

1. C. Ford, Some results on the Schur index of a representation of a finite group, Canad. J. Math. 22 (1970), 626-640. MR 41 \#5511.

2. M. Benard and M. Schacher, The Schur subgroup. II (to appear).

3. E. Witt, Die algebraische Strukture des Gruppenringes einer endlichen Gruppe über einem Zahlkörper, J. Reine Angew. Math. 190 (1952), 231-245. MR 14, 845.

Department of Mathematics, University of Illinois, Urbana, Illinois 61801 